\title{
Virtually Isotropic Transmission Media with Fiber Raman Amplifier
}

\author{
Sergey Sergeyev, Sergei Popov, Ari T. Friberg
}

\begin{abstract}
We report a theoretical study and simulations of a novel fiber-spin tailoring technique to suppress the polarization impairments, namely polarization mode dispersion (PMD) and polarization dependent gain (PDG), in fiber Raman amplifiers. Whereas use of depolarizer or multiplexing pump laser diodes with a final degree of pump polarization of $1 \%$ for periodically spun fiber results in PDG of about $0.3 \mathrm{~dB}$, we demonstrate that application of just a two-section fiber (where the first part is short and has no spin, and the second one is periodically spun) can reduce the PDG to as low as below $0.1 \mathrm{~dB}$.
\end{abstract}

Index Terms - Optical fiber amplifiers, Raman scattering.

\section{INTRODUCTION}

$\mathrm{P}$ olarization impairments are among major factors limiting the progress in further increase of transmission rates and overall capacity of the next generation of optical networks based on distributed fiber Raman amplification. For example, application of ultra-long $120 \mathrm{~km}$ fiber Raman laser creates broadband quasi-lossless fiber spans with simultaneous spatial and spectral transparency $[1,2]$. However, further application of this technology for ultra-high bit-rate communication systems requires the design of quasi-isotropic media, i.e., addressing the issue of polarization impairments in a form of polarization mode dispersion (PMD) and Raman polarization dependent gain (PDG). PMD leads to pulse broadening caused by varying group velocities for the pulses with different states of polarization (SOPs) (Fig. 1) [3-8]. PDG is the dependence of Raman gain on the input signal SOP (Fig. 2) [9-15].

By the traditional approach, spinning the fiber periodically, it is possible to reduce PMD to below $0.04 \mathrm{ps} / \mathrm{km}^{1 / 2}$ (Corning LEAF $^{\circledR}$ fiber, manufactured by Corning Inc., USA $\left.[6,7]\right)$, but this is accompanied with a simultaneous increase in Raman PDG [13]. All the existing PDG mitigation schemes are rather expensive (polarization multiplexing of pump laser diodes,

Manuscript received February 17, 2010. This work is supported in part by Ireland Commercialization Fund, under grant CFTD/07/IT/332a.

Sergey Sergeyev is with Waterford Institute of Technology, Cork Road, Waterford, Ireland (corresponding author, phone: 353-5130-2121; e-mail: ssergeyev@wit.ie, sergey.sergeyev@ gmail.com).

Sergei Popov is with Royal Institute of Technology (KTH), Department of Microelectronics and Applied Physics, Electrum 229, SE-164 40 Kista, Sweden (e-mail: sergeip@kth.se).

Ari T. Friberg is with Aalto University, Department of Applied Physics, P.O. Box 13500, FI-00076 Aalto, Finland, University of Joensuu, Department of Physics and Mathematics, P.O. Box 111, FI-80101 Joensuu, Finland, and Royal Institute of Technology (KTH), Department of Microelectronics and Applied Physics, Electrum 229, SE-164 40 Kista, Sweden (email: atf@kth.se). application of a depolarizer) or not very effective (backward pumping) in the case of low PMD fibers $[9,12,16]$.

To develop a reliable technique for simultaneous mitigation of both PMD and PDG, an advanced vector model of a fiber Raman amplifier has to be employed, accounting also for the random birefringence and arbitrary spin profile of the fiber. Sergeyev et al. [13] have recently put forward such a model, which takes the form of ordinary differential equations for the gain of the fiber Raman amplifier as a function of pump and signal states of polarization (SOP), PMD parameter, and the parameters of fiber spinning. Using this model, it was shown that it is possible to mitigate both PDG and PMD by adopting a fiber with a particular spin profile or, more specifically, a two-section fiber ('two-section approach') in which the first section has no spin and the second one is periodically spun [13]. As a result, PDG and PMD in $10 \mathrm{~km}$ of dispersion compensation fiber (DCF) can be suppressed to $0.13 \mathrm{~dB}$ and $0.032 \mathrm{ps} / \mathrm{km}^{1 / 2}$, respectively [13].

To suppress PMD and PDG further, and thus to develop quasi-isotropic transmission media of $50 \mathrm{~km}$ length, we report herein on a novel approach to optimize the parameters of the first section of the fiber, viz., its correlation length and PMD. In addition, we compare our new technique with the generic method of PDG suppression that is based on the application of a depolarizer $[9,16]$.

\section{OPTIMIZATION OF PARAMETERS FOR 'TWO-SECTION APPROACH'}

For a high PMD fiber, for example a polarization maintaining (PM) fiber, if the pump wave is polarized along one of the birefringence axes, the parallel orientation of the signal corresponds to maximum Raman gain, while the orthogonal orientation results in minimum gain [11]. The difference between two gains (in $\mathrm{dB}$ units) is a quantitative measure of polarization dependent gain which takes the maximum value for this case [11]. If initially the pump field is equally shared between the two orthogonal states of polarization, the pump SOP evolves through all the possible polarization states and returns to its original state after a beat length $L_{\mathrm{b}}$. On average, the pump power is the same along both orthogonal axes. Hence, there is no difference in the Raman gain values and the PDG takes on the minimum value which equals zero [11]. Unlike a PM fiber, the random birefringence in a single mode fiber can be represented in terms of a fixed modulus model (FMM), where the length of the birefringence vector is fixed and its orientation is driven by a white-noise process, and a more complex random modulus model (RMM), in which both 
the orientation and the length of the birefringence vector

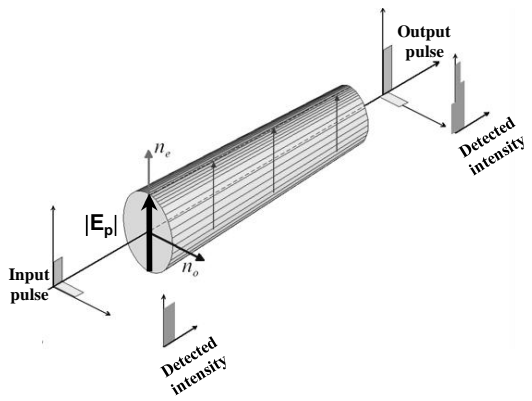

a)

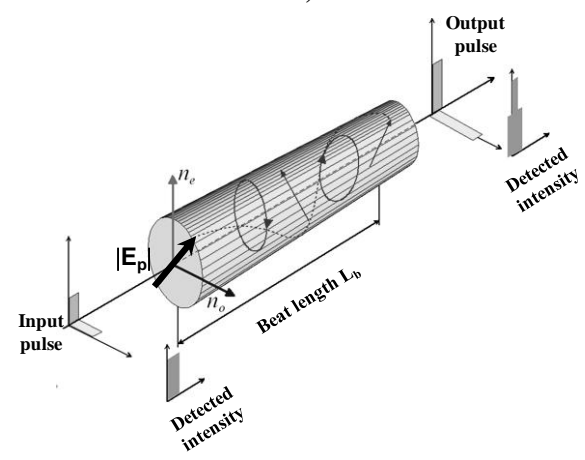

b)

Figure 1. Optical pulse distortion in terms of differential group delay DGD, caused by polarization mode dispersion PMD and polarization dependent gain PDG, in a Raman amplifier with fixed birefringence, e.g., a polarization maintaining (PM) fiber. (a) Pump electric-field vector is oriented along a birefringence axis; (b) pump electric-field is equally shared between the two orthogonal states of polarization.

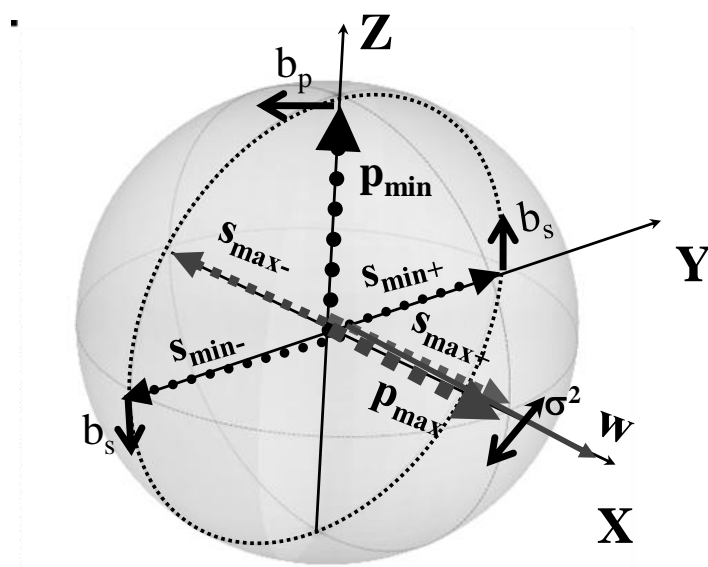

Figure 2. Evolution of the pump (p) and signal (s) states of polarization on the Poincaré sphere, as well as the rotation of the local birefringence vector $\mathbf{w}$. Vectors $\mathbf{p}$ and $\mathbf{s}$ rotate around the local axis $\mathbf{w}$ at rates $b_{\mathrm{p}}$ and $b_{\mathrm{s}}$, while vector $\mathbf{w}$ rotates randomly in the equatorial plane at the rate $\sigma=L_{\mathrm{c}}^{-1 / 2}\left(L_{\mathrm{c}}\right.$ is the correlation length). Maximum PDG: initial orientations of the pump SOP along the PSP, i.e., $\mathbf{p}_{\max }=(1,0,0)$, signal polarizations $\mathbf{s}_{\max +}=(1,0,0)$ giving the maximum Raman gain, and $\mathbf{s}_{\max }=(-1,0,0)$ the minimum gain. Minimum PDG: pump power is equally shared between PSPs, for example $\mathbf{p}_{\min }=(0,0,1)$, signal polarizations $\mathbf{s}_{\min +}=(0,10)$ leading to the maximum Raman gain, and $\mathbf{s}_{\min }=(0,-1,0)$ to the minimum gain. These orientations correspond to the minimum polarization dependent gain (PDG) for the case of oscillatory behavior of the pump to signal SOP projection, i.e., when $b_{\mathrm{p}}-b_{\mathrm{s}}$ is much higher than the de-correlation rate $L_{\mathrm{c}}^{-I}$. change randomly [13]. Poole and Wagner [3] have shown that the so-called principal states of polarization (PSPs) are a key concept in analyzing PMD in fibers with randomly varying birefringence. The PSPs correspond to two orthogonal states of polarization at the input that yield polarization states that are independent of frequency to first order at the output. For a PM fiber, the PSPs coincide with the fast and slow axes and the input and output PSPs are the same. For a fiber with random birefringence (SM fiber), the input and output PSPs are different. In view of this, we demonstrated experimentally and theoretically in our previous publications that, similar to PM fibers, PDG in SM fibers takes on its maximum value if the pump SOP is oriented along a PSP, and a small minimum one (different from zero) if the pump SOP is equally shared initially between the PSPs [12-15].

However, a SM fiber with PMD suppressed by periodic spinning becomes isotropic, i.e., without absolute anisotropy axis with reference to the whole fiber. As a result, the Raman gain depends only on the relative orientation between the input signal and the pump SOPs, which is not changing along the length of the isotropic fiber: co-polarized pump and signal waves give the upper limit of maximum Raman gain and cross-polarized waves result in the lower limit of minimum gain as a function of the PMD value. Finally, maximum and minimum PDGs converge and approach the upper limit of PDG which can exceed $20 \mathrm{~dB}$ [13].

The vector model describing the polarization dependence of the Raman gain in fibers with random or regular birefringence can instructively be demonstrated using the Poincaré sphere. Here, the states of the signal and pump polarization are considered in terms of vectors $\boldsymbol{s}=\left(s_{1}, s_{2}, s_{3}\right)$ and $\boldsymbol{p}=\left(p_{1}, p_{2}, p_{3}\right)$ pointing to positions on the Poincaré sphere (Fig. 2). Fiber birefringence can be presented as a rotation of the $\boldsymbol{s}$ and $\boldsymbol{p}$ vectors on the Poincaré sphere around the birefringence vector $\mathbf{w}$, which, in turn, oscillates regularly due to the periodic fiber spinning or/and randomly due to random birefringence. These regular or random oscillations of the birefringence vector lead to the slowing down of the signal SOP's rotation, i.e., to a suppression of PMD.

As is illustrated in Fig. 2, the signal and pump states of polarization revolve on the Poincare sphere in the same direction but at different rates $b_{\mathrm{s}}$ and $b_{\mathrm{p}}\left(b_{i}=\pi / L_{\mathrm{b} i}\right.$ is the birefringence strength, $L_{\mathrm{b} i}$ is the beat length) around the birefringence vector $\mathbf{w}$. If the difference $b_{\mathrm{p}}-b_{\mathrm{s}}$ is much higher than the de-correlation rate $1 / L_{c}$, then $s$ and $\boldsymbol{p}$ vectors reach mutually parallel and orthogonal orientations, and oscillatory behavior occurs for the averaged projection of the signal SOP on the pump SOP, i.e., for $\langle x\rangle=\langle\boldsymbol{s} \cdot \boldsymbol{p}\rangle$ [14]. As a result, the projections corresponding to the max/min Raman gain, i.e., $\left\langle x_{\max }\right\rangle$ and $\left\langle x_{\min }\right\rangle$, oscillate in anti-phase along the fiber and merge at distances of $z_{n}=n T / 2$, where $T$ is the spatial period, and $n$ is an integer [14]. It is quite clear that for a low PMD fiber such a rotation is absent. This fiber can be periodically spun with the spinning profile (in units of rad)

$A(z)=A_{0} \sin \frac{2 \pi z}{p}$,

where $A_{0}$ and $p$ are the spinning amplitude and period, and $z$ is a distance along the fiber. Therefore, if we combine a short- 
length fiber $\left(L_{1}=z_{1}\right)$ without spin with a long periodically spun fiber with the length of $L_{2}>>L_{1}$, the projections will be the same from the point of merging to the end of the fiber. In this case, as shown in [13], PDG is approximately equal to $\left\langle x_{\max }\right\rangle-\left\langle x_{\min }\right\rangle$ averaged over the length of the fiber. This means that the PDG depends on the parameters of the fiber's first section only and can be minimized [13]. The minimum required length of the first section of fiber $L_{l}$ has been found as follows [13]

$$
L_{1} \approx 2 \pi L_{c} / \sqrt{4\left[\pi D_{p}^{(1, u n)} c \sqrt{2 L_{c}}\left(1 / \lambda_{p}-1 / \lambda_{s}\right)\right]^{2}-1 / 4}
$$

where $\lambda_{\mathrm{p}}$ and $\lambda_{\mathrm{s}}$ are the pump and signal wavelengths, $D_{\mathrm{p}}{ }^{(1, u n)}$ is the PMD parameter of the first section of the fiber, and $L_{\mathrm{c}}$ is the correlation length [13]. For a two-section fiber, the meansquare differential group delay (DGD) $\sqrt{\left\langle\Delta \tau_{t s}^{2}\right\rangle}$ depends on the DGDs of the fiber without spin $\left\langle\Delta \tau_{u n}^{2}\right\rangle$ and the periodically spun fiber $\left\langle\Delta \tau_{p s}^{2}\right\rangle$ as follows

$$
\sqrt{\left\langle\Delta \tau_{t s}^{2}(L)\right\rangle}=\sqrt{\left\langle\Delta \tau_{u n}^{2}\left(L_{1}\right)\right\rangle+\left\langle\Delta \tau_{p s}^{2}\left(L_{2}\right)\right\rangle} .
$$

The spin induced reduction factor (SIRF) is defined as [5]

$$
\operatorname{SIRF}=\frac{\sqrt{\left\langle\Delta \tau_{t s}^{2}(L)\right\rangle}}{\left\langle\Delta \tau_{u n}^{2}(L)\right\rangle}=\frac{D_{p}^{t s}(L)}{D_{p}^{u n}(L)}
$$

where $\left\langle\Delta \tau_{u n}^{2}(L)\right\rangle=D_{p}^{(u n)} \sqrt{\left(\exp \left(-L / L_{c}\right)-1\right) L_{c}+L}$, and $D_{p}^{(u n)}, D_{p}^{(t s)}$ are the PMD parameters for the fiber without spinning and the two-section fiber [13]. If the initial (before spinning) PMD for the periodically spun fiber coincides with the PMD of the first fiber section, i.e., $D_{p}^{(2, s p)}=D_{p}^{(1, u n)}$, and if $L \gg L_{c}$ and $\left\langle\Delta \tau_{u n}^{2}\left(L_{1}\right)\right\rangle \gg\left\langle\Delta \tau_{p s}^{2}\left(L_{2}\right)\right\rangle$, the SIRF for the twosection section fiber can be calculated as [13]

$$
S I R F=\sqrt{\left(\exp \left(-L_{1} / L_{c}\right)-1\right) L_{c} / L+L_{1} / L} .
$$

Otherwise, the SIRF is calculated as follows [5]

$$
\begin{gathered}
\frac{d S I R F^{2}}{d z^{\prime}}=\epsilon_{1}\left\langle\widehat{\Omega}_{1}\right\rangle, \\
\frac{d\left\langle\hat{\Omega}_{1}\right\rangle}{d z^{\prime}}=-\left\langle\hat{\Omega}_{1}\right\rangle+2 \alpha\left(z^{\prime}\right) L_{c}\left\langle\hat{\Omega}_{2}\right\rangle+1, \\
\frac{d\left\langle\hat{\Omega}_{2}\right\rangle}{d z^{\prime}}=-2 \alpha\left(z^{\prime}\right) L_{c}\left\langle\hat{\Omega}_{1}\right\rangle-\left\langle\hat{\Omega}_{2}\right\rangle-\epsilon_{2}\left\langle\widehat{\Omega}_{3}\right\rangle, \\
\frac{d\left\langle\hat{\Omega}_{3}\right\rangle}{d z^{\prime}}=\epsilon_{2}\left\langle\hat{\Omega}_{2}\right\rangle .
\end{gathered}
$$

Here $z^{\prime}=z / L_{c},\left\langle\widehat{\Omega}_{i}\right\rangle(i=1,2,3)$ are the averaged and normalized components of the PMD vector, $\epsilon_{1}=L_{c} / L, \epsilon_{2}=\left(2 \pi L_{c}\right) / L_{b}$, and $\alpha\left(z^{\prime}\right)=\partial A\left(z^{\prime}\right) / \partial z^{\prime}$ is the spin rate.
It is well known that the SIRF for the periodic spin rate can reach the minimum value of less than 0.01 for the phasematching condition $A_{0} \approx 1.2$ [5]. It means that Eq. (5) along with Eq. (2) can be used for optimization of the first section of the fiber in the context of parameters $L_{c}, L_{l}$, and $D_{p}^{(I, u n)}$. As a result, we obtain

$$
\sqrt{L_{c}} D_{p}^{(1, u n)}=\frac{1}{4 \pi\left(1 / \lambda_{p}-1 / \lambda_{S}\right)}
$$

\section{TWO-SECTION APPROACH VS DEPOLARIZER APPLICATION FOR MITIGATION OF PMD AND PDG}

To justify the method of the first section's optimization, we have used Eqs. (6) to find the SIRF and the advanced model of the fiber Raman amplifier for a two-section fiber [13]. We also have used both models to calculate PDG and PMD in the case when a depolarizer is applied.

It is known from experimental measurements that the PDG for backward pumping is significantly lower than for forward pumping, while the average gain is higher $[9,12]$. Hence, to consider the more instructive and practical example, here we deal with forward pumping. We consider also a two-section fiber where the first section of length $L_{l}$ has no spin and the second one of length $L_{2}$ is periodically spun. In our approach, we make use of the FMM, which is simpler but nonetheless demonstrates results for the SIRF similar to those obtained with the RMM for the case $\left(p / L_{b}\right)^{2} \ll 1$ [8].

We further neglect both pump depletion and the signalinduced cross-phase modulation (XPM), which is valid when the pump power is much larger than the signal power. Additionally, the pump induced XPM, i.e., term $\varepsilon_{s p} \boldsymbol{P} \times \boldsymbol{S}$, is eliminated by a specific transformation [10]. It follows that PDG is a function of the length of the signal Stokes vector, which is invariant under transformations such as the one employed in [10]. Thus, nonlinear phase rotation caused by pump-induced XPM has no affect on polarization dependent gain [10]. Next, we choose the reference frame in the Stokes space in such a way that the $x$ axis coincides with the input principal state of polarization. In view of notations in Fig. 2, there are two pump SOPs for which PDG take the maximum and minimum value. The first SOP is oriented along PSP, i.e., $\boldsymbol{p}_{\max }=(1,0,0)$, while the second one is arbitrary, for example $\boldsymbol{p}_{\min }=(0,0,1)$, which provides equal sharing of the pump power between the two PSPs [13-15]. As follows from [10, 13-15], the max/min of PDG can be calculated as

$$
P D G=10 \log \left(\frac{\left\langle s_{0}^{\max }\right\rangle}{\left\langle s_{0}^{\min }\right\rangle}\right) .
$$

Here $<\ldots>$ denotes averaging over the birefringence fluctuations along the fiber.

The averaged length of the Poincaré vector $\left\langle s_{0}\right\rangle$ at the fiber output is a function of the input pump and the signal SOPs and can be found from [12]:

$$
\begin{gathered}
\frac{d\left\langle s_{0}\right\rangle}{d z^{\prime}}=\varepsilon_{1} \exp \left(-\varepsilon_{2} z^{\prime}\right)\langle x\rangle, \\
\frac{d\langle x\rangle}{d z^{\prime}}=\varepsilon_{1} \exp \left(-\varepsilon_{2} z^{\prime}\right)\left\langle s_{0}\right\rangle-\varepsilon_{3}\langle y\rangle,
\end{gathered}
$$




$$
\begin{gathered}
\frac{d\langle\mathrm{y}\rangle}{d z^{\prime}}=\varepsilon_{3}\left[\langle x\rangle-\tilde{p}_{1}(0) \tilde{s}_{1}(0) \exp \left(-z^{\prime}\right)\right]-2 \alpha\left(z^{\prime}\right) L_{c}\langle u\rangle-\frac{\langle y\rangle}{2}, \\
\frac{d\langle u\rangle}{d z^{\prime}}=2 \alpha\left(z^{\prime}\right) L_{c}\langle y\rangle-\frac{\langle u\rangle}{2} .
\end{gathered}
$$

Here $z^{\prime}=z / L_{c},\langle x\rangle=\left\langle\tilde{p}_{1} \tilde{s}_{1}+\tilde{p}_{2} \tilde{s}_{2}+\tilde{p}_{3} \tilde{s}_{3}\right\rangle$ is the projection of the signal SOP onto the pump SOP, $\langle y\rangle=\left\langle\tilde{p}_{3} \tilde{s}_{2}-\tilde{p}_{2} \tilde{s}_{3}\right\rangle$, $\langle u\rangle=\left\langle\tilde{p}_{3} \tilde{s}_{1}-\tilde{p}_{1} \tilde{s}_{3}\right\rangle, \quad \varepsilon_{1}=g P_{i n} L_{c} / 2, \varepsilon_{2}=\alpha_{s} L_{c}, \quad \varepsilon_{3}=$ $\pi D_{p} c \sqrt{2 L_{c}}\left(1 / \lambda_{p}-1 / \lambda_{s}\right), g$ is the Raman gain coefficient, $P_{i n}$ is the input pump power, and $\alpha_{\mathrm{s}}$ and $\alpha_{\mathrm{p}}$ are the signal and pump losses, respectively. Here $\widetilde{\boldsymbol{p}}$ and $\tilde{\boldsymbol{s}}$ are the pump and signal SOPs in the reference frame in which $\widetilde{\boldsymbol{W}}=\left(2 b_{i}, 0,0\right)$, i.e., the birefringence vector is oriented along PSP on the Poincaré sphere.

To justify the validity of FMM application we calculated the beat length as follows [4]:

$$
L_{b}=\frac{\lambda_{s} \sqrt{2 L_{c}}}{D_{p} c}
$$

and chose the fiber spinning amplitude to satisfy the relation $\left(p / L_{b}\right)^{2} \ll 1$. To prove the feasibility of the two-section approach, we further calculated mean-square gain fluctuations (MSGF) of the two-section fiber $\left\langle\sigma_{G}^{2}(L)\right\rangle$ as follows

$$
\left\langle\sigma_{G}^{2}(L)\right\rangle=\sqrt{\left\langle\sigma_{G}^{2}\left(L_{1}\right)\right\rangle+\left\langle\sigma_{G}^{2}\left(L_{2}\right)\right\rangle},
$$

where $\left\langle\sigma_{G}^{2}\left(L_{1}\right)\right\rangle$ and $\left\langle\sigma_{G}^{2}\left(L_{2}\right)\right\rangle$ are MSGFs for the first and the second fiber sections. As follows from [10, 15], $\left\langle\sigma_{G}^{2}\left(L_{1}\right)\right\rangle=$ $\left(\left\langle s_{0}^{2}\left(L_{1}\right)\right\rangle-\left\langle s_{0}\left(L_{1}\right)\right\rangle^{2}\right) /\left\langle s_{0}\left(L_{1}\right)\right\rangle^{2}$ and $\left\langle s_{0}\left(L_{1}\right)\right\rangle^{2}$ is found from Eqs. (8) for $\alpha\left(z^{\prime}\right)=0$ and $\left\langle s_{0}^{2}\left(L_{1}\right)\right\rangle$ is obtained from [15]

$$
\begin{gathered}
\frac{d\left\langle s_{0}^{2}\right\rangle}{d z^{\prime}}=2 \varepsilon_{1} \exp \left(-\varepsilon_{2} z^{\prime}\right)\left\langle s_{0} x\right\rangle, \\
\frac{d\left\langle s_{0} x\right\rangle}{d z^{\prime}}=\varepsilon_{1} \exp \left(-\varepsilon_{2} z^{\prime}\right)\left(\left\langle s_{0}^{2}\right\rangle+\left\langle x^{2}\right\rangle\right)-\varepsilon_{3}\left\langle s_{0} y\right\rangle, \\
\frac{d\left\langle x^{2}\right\rangle}{d z^{\prime}}=2 \varepsilon_{1} \exp \left(-\varepsilon_{2} z^{\prime}\right)\left\langle s_{0} x\right\rangle-2 \varepsilon_{3}\langle x y\rangle, \\
\frac{d\left\langle s_{0} y\right\rangle}{d z^{\prime}}=\varepsilon_{1} \exp \left(-\varepsilon_{2} z^{\prime}\right)\langle x y\rangle+\varepsilon_{3}\left[\left\langle s_{0} x\right\rangle\right. \\
\left.-\left\langle s_{0}\right) \tilde{p}_{1}(0) \tilde{s}_{1}(0) \exp \left(-z^{\prime}\right)\right]-\frac{\left\langle s_{0} y\right\rangle}{2}, \\
\frac{d\langle x y\rangle}{d z^{\prime}}=\varepsilon_{1} \exp \left(-\varepsilon_{2} z^{\prime}\right)\left\langle s_{0} y\right\rangle+\varepsilon_{3}\left[\left\langle x^{2}\right\rangle-\left\langle y^{2}\right\rangle\right. \\
\left.-\langle x) \tilde{p}_{1}(0) \tilde{s}_{1}(0) \exp \left(-z^{\prime}\right)\right]-\frac{\langle x y\rangle}{2},
\end{gathered}
$$

$$
\begin{gathered}
\frac{d\left\langle y^{2}\right\rangle}{d z^{\prime}}=2 \varepsilon_{3}\left[\langle x y\rangle-\left\langle y^{2}\right\rangle-\langle y\rangle \tilde{p}_{1}(0) \tilde{s}_{1}(0) \exp \left(-z^{\prime}\right)\right] \\
-\left(\left\langle y^{2}\right\rangle-\left\langle u^{2}\right\rangle\right) \\
\frac{d\left\langle u^{2}\right\rangle}{d z^{\prime}}=\left\langle y^{2}\right\rangle-\left\langle u^{2}\right\rangle .
\end{gathered}
$$

Keeping in mind that $\alpha\left(z^{\prime}\right) \neq 0,\left\langle\sigma_{G}^{2}\left(L_{2}\right)\right\rangle$ cannot be found in the same way as $\left\langle\sigma_{G}^{2}\left(L_{1}\right)\right\rangle$, i.e., with the help of Eqs. (10). However, it was shown by Bettini et al. [17] for the case of Raman amplification in unidirectionally spun fibers that the maximum for the mean-square gain fluctuations is shifted to higher PMD values with increased fiber spinning frequency. It means that the gain fluctuations will be much lower for periodically spun fiber at the phase-matching condition $A_{0} \approx$ 1.2 than calculated from Eqs. (12).

Lin and Agrawal [10] demonstrated that the application of depolarizer results in transformation of coefficient $\varepsilon_{1}$ in Eqs. (9) and (12) as follows: $\varepsilon_{1} \rightarrow \varepsilon_{1} D O P$, where DOP is a degree of polarization at the depolarizer output.

\section{RESULTS AND DISCUSSION}

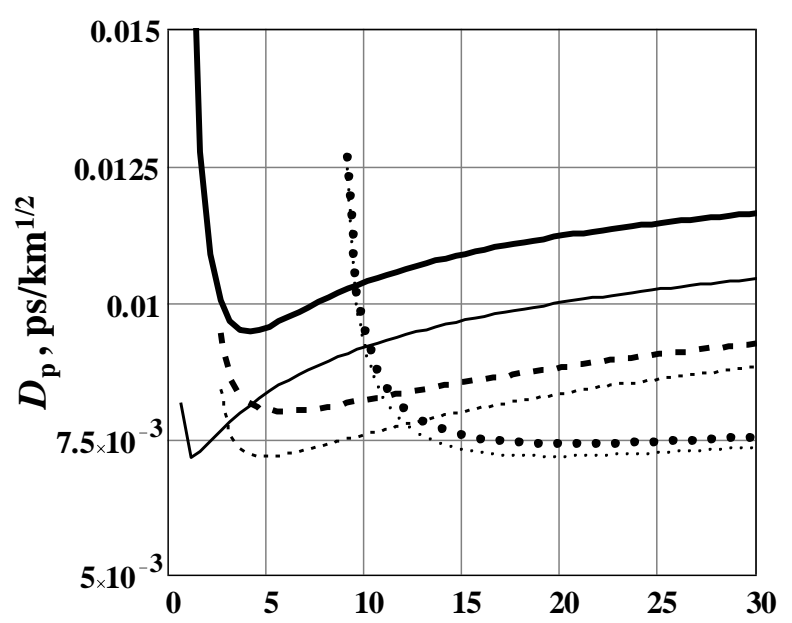

Correlation length $L_{\mathrm{c}}, \mathrm{m}$

Figure 3. PMD parameter $D_{p}$ as a function of correlation length $L_{c}$ in the two-section fiber according to Eqs. $(1,3,4-6)$ for $D_{p}^{(l)}=0.05 \mathrm{ps} \cdot \mathrm{km}^{-1 / 2}$ (solid line), $D_{p}^{(1)}=0.1 \mathrm{ps} \cdot \mathrm{km}^{-1 / 2}$ (dotted line), and $D_{p}^{(1)}=0.2 \mathrm{ps} \cdot \mathrm{km}^{-1 / 2}$ (dashed line), calculated with the help of Eqs. $(1,3,4-6)$ (thin lines) and with the help of Eqs. (1,3,5-6) (thick lines). Parameters: $L=50 \mathrm{~km}, \alpha_{\mathrm{s}}=0.2$ $\mathrm{dB} / \mathrm{km}, \lambda_{\mathrm{p}}=1460 \mathrm{~nm}, \lambda_{\mathrm{s}}=1550 \mathrm{~nm}, g=2.3 \mathrm{~W}^{-1} \mathrm{~km}^{-1}, P=0.5 \mathrm{~W}$.

First, we found the PMD value $D_{p}^{(1, u n)}$, correlation length $L_{\mathrm{c}}$, and the length $L_{1}$ for the first section from Eqs. (7) and (2) to provide minimum PMD value for the two-section fiber. We have used parameters typical for a single-mode-fiber based distributed fiber Raman amplifier: $L=50 \mathrm{~km}, \alpha_{\mathrm{s}}=0.2 \mathrm{~dB} / \mathrm{km}$, $\lambda_{\mathrm{p}}=1460 \mathrm{~nm}, \lambda_{\mathrm{s}}=1550 \mathrm{~nm}, g=2.3 \mathrm{~dB} \mathrm{~W}^{-1} \mathrm{~km}^{-1}$, and $P=0.5 \mathrm{~W}$. Next, we calculated PMD values for the two-section fiber with the help of Eqs. (5) and (6) and accounting for Eq. (4). The results are shown in Fig. 3. In view of Eq. (7), one of the 
parameters $D_{p}^{(1, u n)}$ or $L_{\mathrm{c}}$ can be chosen arbitrary, so we picked $D_{\mathrm{p}}{ }^{(1)}=0.05,0.1$ and $0.2 \mathrm{ps} \cdot \mathrm{km}^{-1 / 2}$ (Fig. 3). As is seen from Fig. 3 , Eqs. (2) and (7) can be used for the calculation of the parameters for the first part of the two-section fiber,

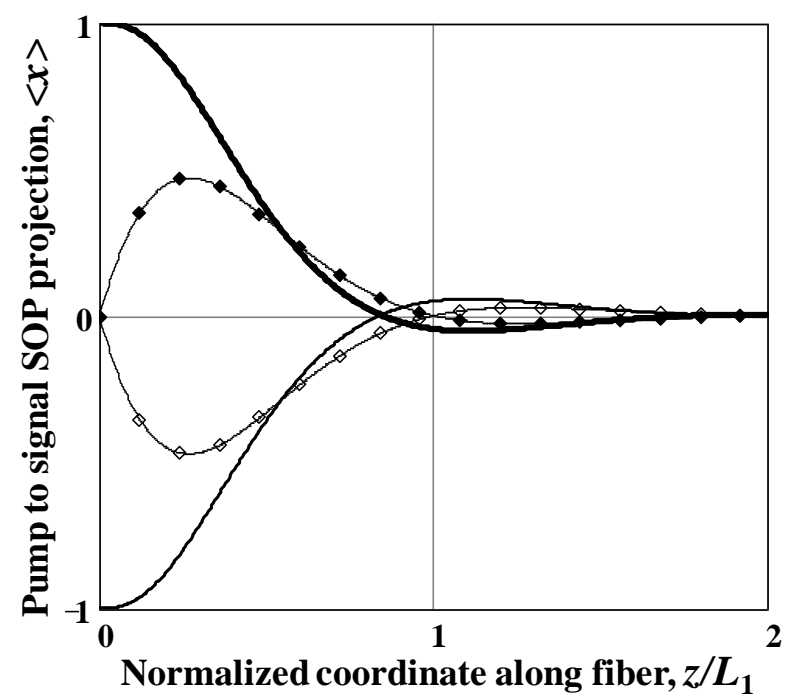

Figure 4. Evolution along the fiber length of the signal-to-pump SOP projection $\langle\mathrm{x}\rangle$ for the case of the fiber without spinning. Minimum PDG: empty and filled diamonds; maximum PDG: thick and thin solid lines. Projections for the maximum gain: thick solid line, filled diamonds; for the minimum gain: thin solid line, empty diamonds. Parameters: $D_{p}^{(l)}=$ $0.1 \mathrm{ps} \cdot \mathrm{km}^{-1 / 2}, L_{c}=5 \mathrm{~m}, L=50 \mathrm{~km}, \alpha_{\mathrm{s}}=0.2 \mathrm{~dB} / \mathrm{km}, \lambda_{\mathrm{p}}=1460 \mathrm{~nm}, \lambda_{\mathrm{s}}=$ $1550 \mathrm{~nm}, g=2.3 \mathrm{~W}^{-1} \mathrm{~km}^{-1}, P=0.5 \mathrm{~W}$.

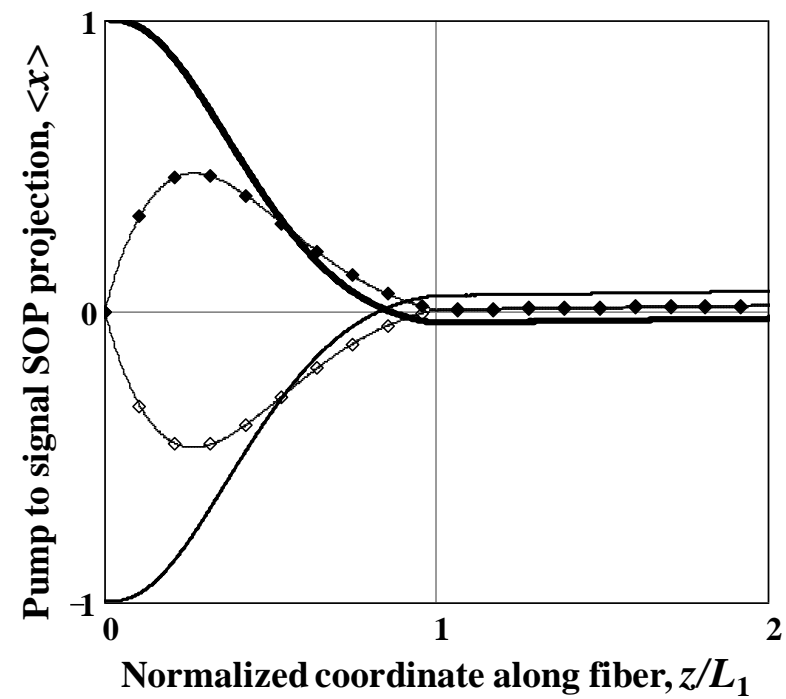

Figure 5. Evolution along the fiber length of the signal-to-pump SOP projection $\langle x\rangle$ for the case of the two-section fiber comprising a fiber without spin and a periodically spun fiber (dashed line). Minimum PDG: empty and filled diamonds; maximum PDG: thick and thin solid lines. Projections for the maximum gain: thick solid line, filled diamonds; for the minimum gain: thin solid line, empty diamonds. Parameters: $D_{p}^{(1)}=0.1 \mathrm{ps} \cdot \mathrm{km}^{-1 / 2}, L_{c}=5 \mathrm{~m}, L_{l} \approx$ $56.25 \mathrm{~m}, L=50 \mathrm{~km}, \alpha_{s}=0.2 \mathrm{~dB} / \mathrm{km}, \lambda_{p}=1460 \mathrm{~nm}, \lambda_{s}=1550 \mathrm{~nm}, g=2.3 \mathrm{~dB}$ $\mathrm{W}^{-1} \mathrm{~km}^{-1}, P=0.5 \mathrm{~W}, p=0.51 \mathrm{~m}$.

which leads to the minimum PMD value in the two-section fiber. As a result, we obtained optimal parameters for the first section of the fiber as $D_{\mathrm{p}}{ }^{(1)}=0.1 \mathrm{ps} \cdot \mathrm{km}^{-1 / 2}, L_{\mathrm{c}}=5 \mathrm{~m}, L_{l} \approx 56.25$ $\mathrm{m}$ (dashed line in Fig. 3). We use these parameters to calculate the evolution of pump-to-signal SOP projection $\langle x\rangle$ along the fiber without spinning (Fig. 4).

As follows from Fig. 4, the length at which the projections merge depends on the input pump SOP: it coincides with the optimal length of fiber $L_{1}$ for the pump equally shared between the PSPs at the input, and it is slightly less than $L_{l}$ for the input pump SOP oriented along the PSP. Thus, the projections will be the same from the input to the output of the second section of the fiber only for minimum PDG (Fig. 5). For the pump SOP providing maximum PDG, projections corresponding to the maximum and minimum gain $\left\langle x_{\max }\right\rangle$ and $\left\langle x_{\min }\right\rangle$ are swapped for the second section (Fig. 5). In view of $P D G \approx\left\langle x_{\max }\right\rangle-\left\langle x_{\min }\right\rangle$ and the different dependence of $\left\langle x_{\max }\right\rangle$ and $\left\langle x_{\min }\right\rangle$ on spinning amplitude $A_{0}$ and period $p$, PDG can vary from $0.005 \mathrm{~dB}$ to $0.2 \mathrm{~dB}$ for different fiber spinning amplitudes (Fig. 6). We used parameters $D_{\mathrm{p}}^{(1)}=0.1 \mathrm{ps} \cdot \mathrm{km}^{-1 / 2}$, $L_{\mathrm{c}}=5 \mathrm{~m}, L_{l} \approx 62.8 \mathrm{~m}$ and spinning period of $p=0.51 \mathrm{~m}$ in addition to the parameters mentioned above, to calculate PMD and PDG values for the two-section fiber (without spin /periodically spun) as a function of spinning amplitude $A_{0}$ based on Eqs. (6) and (9). The beat length calculated from Eq. (10) results in $L_{b}=2.6 \mathrm{~m}$ and so $\left(p / L_{b}\right)^{2} \ll 1$. Thus, the FMM model of random birefringence is valid for our case. The results are shown in Fig. 6.

Finally, we calculated PMD and maximum/minimum PDG for a periodically spun fiber in the case of application of a depolarizer with degrees of polarization DOP $=1 \%$ and $10 \%$ with the help of Eqs (9) and accounting for changing the parameter in Eqs. (9) as follows: $\varepsilon_{1} \rightarrow \varepsilon_{1} D O P$ (Fig. 6). As follows from [16], temperature fluctuations can lead to an increased DOP for the input pump wave of $10-15 \%$. In addition, the averaged values of DOP can be of $5 \%$ with insertion losses of $1.5 \mathrm{~dB}$ [18]. In view of this, it can lead to an increased PDG value above $1 \mathrm{~dB}$ (Fig. 6) and suppressed averaged gain of $1.5 \mathrm{~dB}$ [18].

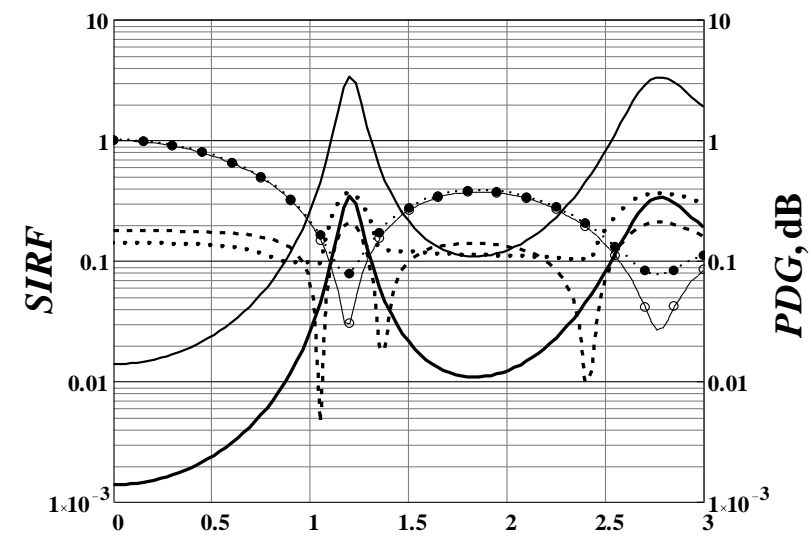

Fiber spinning amplitude $A_{0}$, rad

Fig. 6 SIRF (filled and empty circles) PDG (solid, dotted, and dashed lines) as a function of fiber spinning amplitude $A_{0}$. SIRF for periodically spun fiber: empty circles; two-section fiber: filled circles. PDG for twosection fiber: pump SOP along PSP (dashed line), pump is equally shared between input PSPs (dotted line). PDG for the case of depolarizer application: output DOP for depolarizer 1\% (thin line), output DOP for depolarizer $10 \%$ (thick line). Parameters: $D_{p}{ }^{(l)}=0.1 \mathrm{ps} \cdot \mathrm{km}^{-1 / 2}, L_{c}=5 \mathrm{~m}, L_{l}$ $\approx 56.25 \mathrm{~m}, L=50 \mathrm{~km}, \alpha_{s}=0.2 \mathrm{~dB} / \mathrm{km}, \lambda_{p}=1460 \mathrm{~nm}, \lambda_{s}=1550 \mathrm{~nm}, g=$ $2.3 \mathrm{~dB} \mathrm{~W}^{-1} \mathrm{~km}^{-1} . P=0.5 \mathrm{~W} . p=0.51 \mathrm{~m}$. 
TABLE I

TWO-SECTION APPROACH VS APPLICATION OF DEPOLARIZER

\begin{tabular}{lll}
\hline \hline \multicolumn{1}{c}{ Specifications } & Two-section approach & \multicolumn{1}{c}{$\begin{array}{c}\text { Application of } \\
\text { Depolarizer }\end{array}$} \\
\hline PDG & $0.003-0.3 \mathrm{~dB}$ (Fig. 6) & $0.3-3 \mathrm{~dB}$ (Fig. 6) \\
Min SIRF & 0.007 (Fig. 6) & 0.003 (Fig. 6) \\
Insertion losses & $<0.2 \mathrm{~dB}$ & $1.5 \mathrm{~dB}[18]$ \\
Mean-square gain & $2-6 \%$ & $0.4-0.6 \%$ \\
fluctuations & &
\end{tabular}

${ }^{\mathrm{a}} P D G \geq 1 \mathrm{~dB} \mathrm{~dB}$ for averaged output depolarizer $\mathrm{DOP}=5 \%$ [18].

To complete the comparative analysis of the two-section technique and application of a depolarizer, we calculated also mean-square gain fluctuations for both cases with the help of Eqs. (9), (11), and (12) and notations to Eqs. (12). We find that the signal fluctuations at the input of the first section are negligible and do not exceed of $0.1 \%$, while MSGF for the second section are within 20-60\%. Application of depolarizer with DOP $=10 \%$ shows MSGF within 4-6\%. In view of the notations to Eqs. (12) and the results of [17], MSGF can be suppressed approximately 10 times, i.e., to $2-6 \%$ for the twosection approach and to 0.4-06 \% for the case of depolarizer application, for PMD value of $D_{\mathrm{p}}^{(1)}=0.003 \mathrm{ps} \cdot \mathrm{km}^{-1 / 2}$ (phasematching condition $A_{0} \approx 1.2$ and spinning period of $p=0.5 \mathrm{~m}$ ).

Thus, the suggested cost-effective approach to simultaneous mitigation of PMD and PDG based on two-section (without spin/periodically spun) fibers shows much better results in PDG and insertion losses and slightly worse results for mean square gain fluctuations as compared to the case of depolarizer application. The results of the comparative analysis are summarized in Table I.

\section{CONCLUSION}

In conclusion, we report a cost-effective approach to design a quasi-isotropic transmission medium of $50 \mathrm{~km}$ length with a distributed Raman amplifier which demonstrates much lower polarization dependent gain as compared to the approach of application of a depolarizer.

\section{REFERENCES}

[1] J. D. Ania-Castanon, V. Karalekas, P. Harper, and S. K. Turitsyn, "Simultaneous spatial and spectral transparency in ultralong fiber lasers", Phys. Rev. Lett., vol. 101, 123903-123907 (2008).

[2] S. A. Babin, V. Karalekas, E. V. Podivilov, V. K. Mezentsev, P. Harper, J. D. Ania-Castanon, and S. K. Turitsyn, "Turbulent broadening of optical spectra in ultralong Raman fiber lasers", Phys. Rev. A, vol. 77, 033803-1-5 (2008).

[3] C. D. Poole and R. E. Wagner, "Phenomenological approach to polarization dispersion in long single-mode fibers," Electron. Lett., vol. 22, 1029-1030 (1986).

[4] P. K. A. Wai and C. R. Menyuk, "Polarization mode dispersion, decorrelation and diffusion in optical fibers with randomly varying birefringence", J. Lightwave Technol., vol. 14, 148-157 (1996).

[5] A. Galtarossa, L. Palmieri, A. Pizzinat, B. S. Marks, and C. R. Menyuk, "An analytical formula for the mean differential group delay of randomly-birefringent spun fibers," J. Lightwave Technol., vol. 21, 1635-1643 (2003).

[6] D. A. Nolan, X. Chen, and M.-J. Li, "Fibers with low polarization-mode dispersion", J. Lightwave Technol., vol. 22, 1066-1077 (2004).
[7] M.-J. Li, X. Chen, and D. A. Nolan, "Ultra low PMD fibers by fiber spinning", Optical Fiber Communication Conference (Los Angeles, CA, USA, 2004), paper FA1.

[8] A. Pizzinat, B. S. Marks, L. Palmieri, C. R. Menyuk, and A. Galtarossa, "Influence of the model for random birefringence on the differential group delay of periodically spun fibers," IEEE Photon. Technol. Lett., vol. 15, 819-821 (2003).

[9] M. N. Islam, C. DeWilde, and A. Kuditcher, "Wideband Raman amplifiers", in Raman Amplifiers for Telecommunications 2: SubSystems and Systems, ed. M. N. Islam (Springer, 2004), pp. 445-490.

[10] Q. Lin and G. P. Agrawal, "Vector theory of stimulated Raman scattering and its application to fiber-based Raman amplifiers" J. Opt. Soc. Am. B, vol. 20, 1616-1631 (2003).

[11] R. H. Stolen, "Polarization effects in fiber Raman and Brillouin Lasers", IEEE J. Quant. Electron., vol. 15, 1157-1160 (1979).

[12] S. Popov, S. Sergeyev, and A. T. Friberg, "The impact of pump polarization on the polarization dependence of the Raman gain due to the breaking of a fiber's circular symmetry", J. Opt. A: Pure Appl. Opt. vol. 6, S72-S76 (2004).

[13] S. Sergeyev, S. Popov, A. T. Friberg, "Spun fiber Raman amplifiers with reduced polarization impairments", Opt. Express, vol. 16, 14380-14389 (2008).

[14] S. Sergeyev, S. Popov, and A. T. Friberg, "Modeling polarizationdependent gain in fiber Raman amplifiers with randomly varying birefringence," Opt. Comm., vol. 262, 114-119 (2006).

[15] S. Sergeyev, S. Popov, and A. T. Friberg, "Polarization dependent gain and gain fluctuations in a fiber Raman amplifier", J. Opt. A: Pure Appl. Opt. vol. 9, 1119-1122 (2007).

[16] H. Kazami, S. Matsushita, Y. Emori, T. Murase, M. Tsuyuki, K. Yamamoto, H. Matsuura, S. Namiki, and T. Shiba, "Development of a crystal-type depolarizer", Furakawa Review, vol. 23, 44-47 (2003).

[17] E. Bettini, A. Galtarossa, L. Palmieri, M. Santagiustina, L. Schenato, and L. Ursini, "Polarized backward Raman amplification in unidirectionally spun fibers," IEEE Photon. Tech. Lett., vol. 20, 27-29

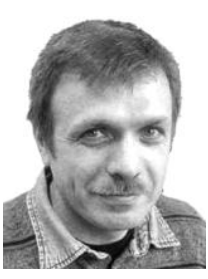
(2008).

[18] http://www.photonic-insight.com/product11.html.

Sergey Sergeyev was born in Nizhny Tagil, Russia, in 1963. He received the M.Sc. and Ph.D. degrees from Belarusian State University (BSU), Minsk, in 1985 and 1991, respectively. He was with the Department of Physics, BSU, from 1985 to 1999 . From 1999 to 2005, he worked in Sweden at Ericsson AB, Royal Institute of Technology, and Acreo AB. Since 2005, he is working at Optics Research Group (ORG), Waterford Institute of Technology, Waterford, Ireland as a Senior Researcher/Project Manager. He has coauthored about 90 journal and conference papers in the areas of fiber optics, laser physics, and spectroscopy.

Sergei Popov received M.Sc. in El. Eng. (1987, Moscow Institute of Physics and Technology) and Comp. Sc. (1989, Air Force Engineering Academy), both in Moscow, Russia. He holds Ph.D. degree in Applied Physics (1998) from Helsinki University of Technology (Espoo, Finland). Dr. Popov pursued his research career in General Physics Institute (Moscow, Russia), Ericsson Telecom AB and Acreo AB (both in Stockholm, Sweden). Currently, he is Associate Professor at Royal Institute of Technology (Stockholm, Sweden); and contributed to over 100 journal and conference papers on laser physics, diffractive and fiber optics, as well as nano-photonics.

Ari T. Friberg holds a PhD in optics from the University of Rochester (1980) and a DSc in applied physics from Helsinki University of Technology (1993). He has held visiting appointments at Imperial College London, Technische Universität Berlin, and Université de Neuchâtel. In 1997 he was appointed Professor at Royal Institute of Technology in Stockholm and since 2007 he has been Academy of Finland Distinguished Professor at Aalto University in Espoo and University of Eastern Finland in Joensuu. He has authored some 200 peer-reviewed journal papers in the areas of diffractive optics, coherence and polarization, electromagnetic optics, near-field optics, and nanophotonics. $\mathrm{He}$ is Past President of the International Commission for Optics (ICO). 